\title{
Invasive Cancer of the Colon with Gastric Metastasis
}

\author{
Antonio Gligorievski* \\ University Clinic of Surgery, The Monastery of Saint Naum, Macedonia
}

*Corresponding author: Antonio Gligorievski, University Clinic of Surgery, The Monastery of Saint Naum, Macedonia.

Received Date: February 26, 2021

Published Date: March 15, 2021

\section{Introduction}

The stomach is a very rare location of metastatic deposits. We present a case of a gastric metastatic deposit from primary colon cancer. Computed tomography (CT) indicated primary colon cancer, but also revealed the presence of cancer in the region of the gastric greater curvature and gastric antrum. With the progression of primary cancer and the creation of an extraluminal tumor mass, the process progresses in the manner that the primary transversal colon tumor merges into the metastatic deposit located in the stomach. The patient underwent subtotal gastrectomy and D2 lymphadenectomy, as well as partial resection of the transversal colon, thus the tumor mass of the stomach and the one in the transversal colon being removed in a single act. Pathohistological analysis of the tumor mass and immunohistochemical tests revealed a primary neoplastic infiltrative process in the colon metastasizing into the stomach.

\section{Introduction}

Bowel cancer is the fourth most common cause of cancer death in the world, and the second most common cause of cancer death in developed countries [1]. The colorectal cancer mortality rate is lowest in Central Africa and Southeast Asia and highest in Central and Eastern Europe [1]. Mortality from colon cancer is age-related. Mortality rates increase with age (1). The prevalence of right bowel cancer increases with increasing patient age [1]. Most colon adenocarcinomas (80\%) occur in existing adenomatous polyps, and progression from a polyploidy adenoma to cancer occurs over several years. Most cancers are asymptomatic until the disease is advanced. The patient may notice blood in the stool, but occult bleeding is more common. Ferro deficiency anemia occurs due to blood loss. The initial diagnosis is usually made by colonoscopy or double-contrast barium enema examination. However, with the increasing use of computed tomography (CT), the initial diagnosis of suspected colon cancer can also be made based on CT findings. Radiographs of the double-contrast barium enema examination usually show ring lesions that are irregular and lead to narrowing of the lumen of the colon. The spread of colon cancer most commonly occurs by the hematogenous route, by the lymphatic route, directly by local invasion of adjacent organs and the peritoneum. Colorectal cancer most often metastasis to the liver, lungs, and peritoneum, but it is also possible for cancer to spread locally, as well as to recur in another part of the colon (metachronous metastases). Rare cases of metastases from colorectal cancer of the spleen, thyroid, esophagus, stomach, urinary tract, and abdominal wall have also been reported in the literature [2]. Stomach metastases from colorectal cancer are extremely rare, 3-4 cases [3-5] have been reported in the literature. We present a case of metastatic gastric cancer, which originates from cancer of the transverse part of the colon.

\section{Presentation of Case}

In February 2016, a 63-year-old man was admitted to our institution due to anemia and suspected bleeding from the digestive tract. The patient complains of difficulty walking and fatigue does not complain of pain in the abdomen but notices that the stool is dark for more than two weeks. The physical 
examination, except for pallor and slight weight loss, revealed no other changes. Preoperative laboratory examination showed Hgb levels. of $79 \mathrm{mmol} / \mathrm{L}$, Er. of 2.64 x 1012/L, Le. of 2.64 x 109/L, HCT of $0.234 / \mathrm{L}$. All other biochemical and hematological tests are normal. Laboratory tests help detect bleeding from the digestive tract. Previously, the patient was treated with anti-ulcer therapy, but after the therapy, the clinical picture did not improve, and the patient left the hospital at his request and come to our clinic for further diagnostic examination and treatment. The patient underwent gastroscopy and an ulcerated infiltration of the gastric wall in the anterior part by a large curvature was detected. A clinical diagnosis of gastric cancer was made and the patient was referred for a further radiological examination of the abdomen. The patient had undergone a CT scan of the abdomen, after intravenous administration of contrast agents. The examination was performed on a GE Bright Speed MDCT computed tomography device with 16 rows of detectors. The CT scan was performed at a tube voltage of $120 \mathrm{KV}$. The rotation speed is $0.8 \mathrm{sec}$. The pitch factor is $1.375: 1$.
The examination was performed in an Auto mA setting. The crosssectional thickness of the native series was $1.5 \mathrm{~mm}$, and that of the post-contrast series was $1.25 \mathrm{~mm}$. The Noise index is 13 . The maximum wide field of view (FOV) was used. The reconstruction matrix was 512 x 512 pixels. Non-ionic, low-osmolar contrast is administered intravenously in a total dose of $100 \mathrm{~mL}$, the rate of contrast application is $3 \mathrm{~mL} / \mathrm{sec}$, and the delay time after contrast application is $25 \mathrm{sec}$. After the end of the examination, an analysis of the obtained scans was made.

In the upper part of the abdomen, there is a soft tissue mass that infiltrates the stomach, but $80 \%$ of the mass is outside the stomach and as we move to the lower part of the abdomen it can be seen that the mass infiltrates the middle part of the transverse colon. A thick and infiltrated wall with a visible ulcer can be seen on the front wall of the stomach by the large curve in the lower part of the corpus and towards the antrum. The TU mass spreads antropyloric as well as to the posterior wall of the stomach. Pediatrically has seen borderline enlarged lymph nodes (Figure 1).
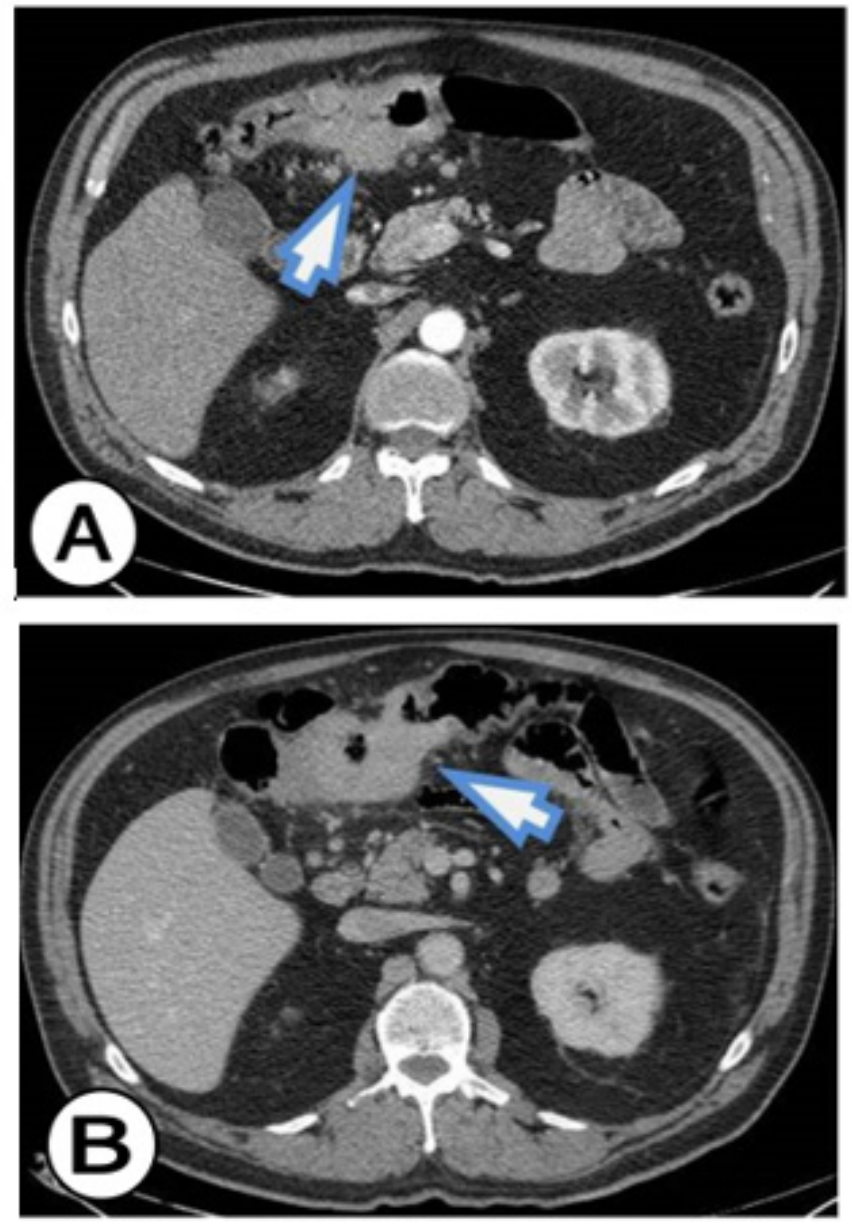

Figure 1: (CT scan from 17.02.2016). Axial CT images of the abdomen at the level of the antral part of the stomach. Axial CT scans show a malignant lesion of the anterior part of the stomach in the area of a large curvature with an exudation (arrow). 
About $80 \%$ of the TU mass is located between the transverse colon and the stomach. The TU mass also infiltrates the wall of the transverse colon where endoluminal masses can be seen. About $90-100 \mathrm{~mm}$ from the length of the transverse colon was with an infiltrated wall, a narrowed lumen, and visible endoluminal TU masses. In the vicinity of the transverse colon, borderline enlarged lymph nodes and a reaction of the surrounding adipose tissue are seen. On the post-contrast series, the tumor showed completely and almost homogeneous contrast enhancement and increases HU. After the analysis of the CT scans, the dilemma remains, whether it is a primary tumor that originates from the stomach and then occupies and infiltrates the transverse colon, or it is a primary tumor of the transverse colon that occupies and infiltrates the stomach. It can also be a gastrointestinal stromal tumor (GIST) that affects and infiltrates the transverse colon and the distal part of the stomach. Our initial conclusion was that it was a GIST that infiltrates the transverse part of the colon and the distal part of the stomach.

Partial resection of the stomach was performed and a TU mass (segment $18 \mathrm{~cm}$ long by the great curvature and $11 \mathrm{~cm}$ by the small curvature) was removed. TU mass was $20 \mathrm{~mm}$ thick and ulcerated. Partial resection Billroth II and a terminal-lateral anastomosis with the jejunum was performed. Also, resection of the transverse colon was performed, TU mass (segment in the length of $14 \mathrm{~cm}$ ) was removed, and terminal-terminal anastomosis was performed. The TUf mass was ulcerated and $9 \mathrm{~cm}$ in length occupies the entire circulatory space of the colon wall. An omentectomy was also performed (on greater omentum $39 \times 18 \times 2 \mathrm{~cm}$ and on lesser omentum $7 \times 4.5 \times 1 \mathrm{~cm}$ ). An oval TU mass with a diameter of 90 $\mathrm{mm}$, which is in a form of a block tumor with the stomach and the transverse colon was removed (Figure 2).
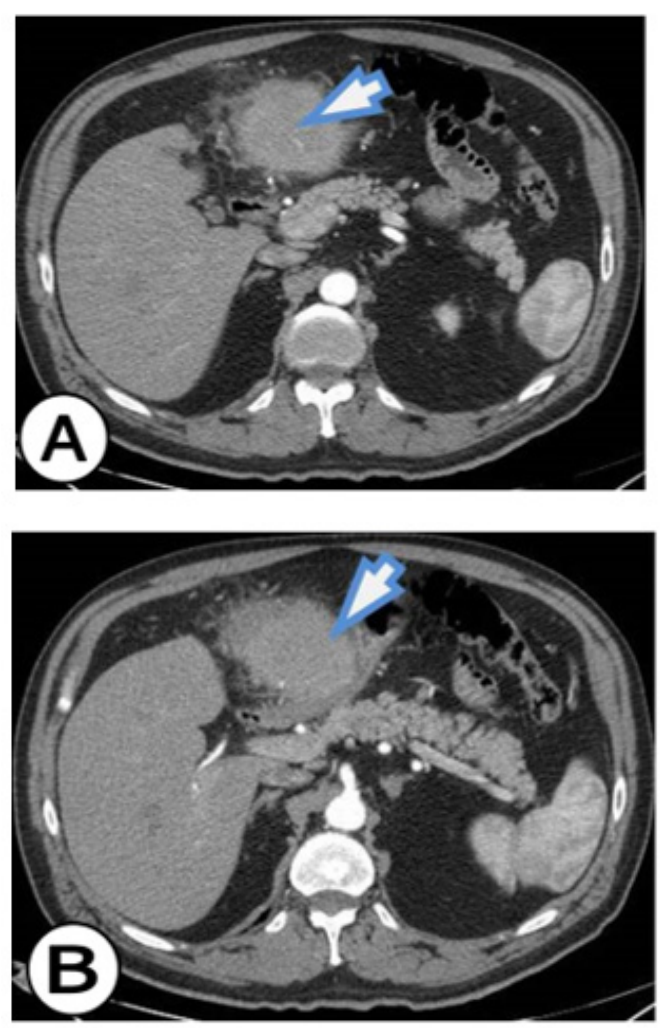

Figure 2: (CT scan from 17.02.2016). Axial CT images of the abdomen above the transverse part of the colon. Axial CT scans show an oval, relatively homogeneous, hyperdense TU mass $80-90 \mathrm{~mm}$ in diameter (arrow).

After the surgery, the patient's son handed us a CD of an examination performed at his father about 7 months ago (on 22.07.2015) when he visited a doctor for the first time due to gait problems. The patient underwent CT angiography, which did not show the significant disease of the arteries of the lower extremities. Seven months after the examination, we performed an analysis of the examination, and the focus of our examination was the shown part of the abdomen on the CT angiography. What we have noticed is an infiltration of the wall of the transverse colon with visible endoluminal TU masses, extraluminal TU masses were not visible. There was a pronounced infiltration of the transverse colon in the length of $92 \mathrm{~mm}$, and the anterior-posterior diameter was 46 $\mathrm{mm}$. Infiltration of the gastric wall into the antropyloric part with volume reduction was also seen. The maximum thickness of the infiltration of the anterior wall of the stomach in the antropyloric region was $14 \mathrm{~mm}$. No evidence of a TU mass connects the stomach 
and colon as it was predominantly noticeable on the CT examination performed after 7 months (on 17.02.2016). With the analysis of CT angiography and the comparison with the CT examination of the abdomen, our opinion is that this is a primary, invasive, malignant neoplasm of the transverse colon that metastasized to the stomach, and in a short time expanded into the omentum and formed block tumor that associates primary colon cancer with metastatic gastric deposit. This opinion stems from the fact that the TU of the colon is much larger in volume than the TU of the stomach, but we cannot rule out with certainty that it is the synchronous occurrence of adenocarcinoma of the colon and adenocarcinoma of the stomach (Figure 3).

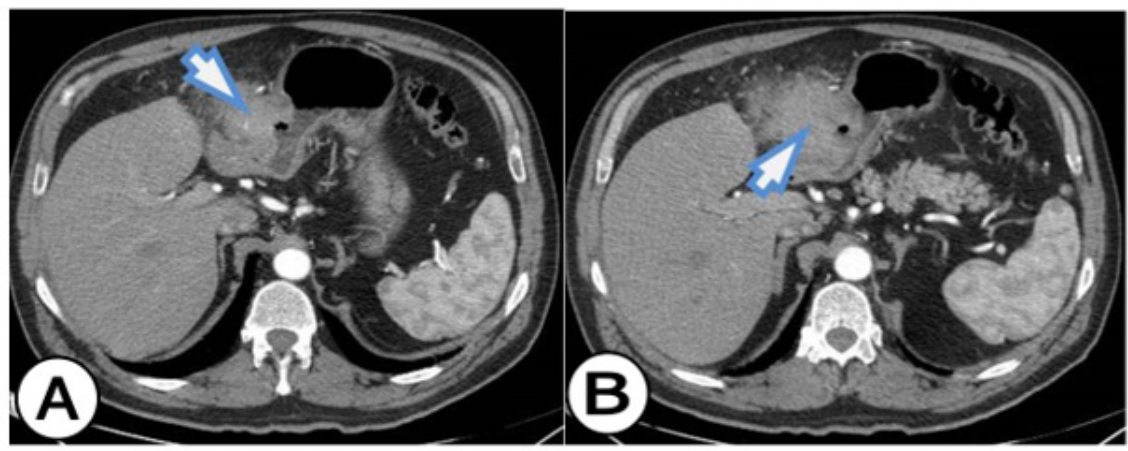

Figure 3: (CT scan from 17.02.2016). Axial CT images of the abdomen at the height of the transverse part of the colon. Axial CT scan shows a malignant lesion of the transverse colon with narrowing of the lumen (arrow).

After the pathohistological analysis of the removed abdominal TU mass and the performed immunohistochemically examinations with CK20 which is positive for the tumor population and CK7 which is negative for the tumor population, a final pathohistological diagnosis is made for primary cancer of the colon with metastasis. According to the morphological appearance and immunohistochemically profile, it is suitable for adenocarcinoma of the colon with a direct spread in the omentum and stomach with the following characteristics according to AJCC 2010 - pTNM - pT4b, pN0, pMx, pL0, G2, NG2 Stage IIC. Dukes B. MAC B3.

\section{Discussion}
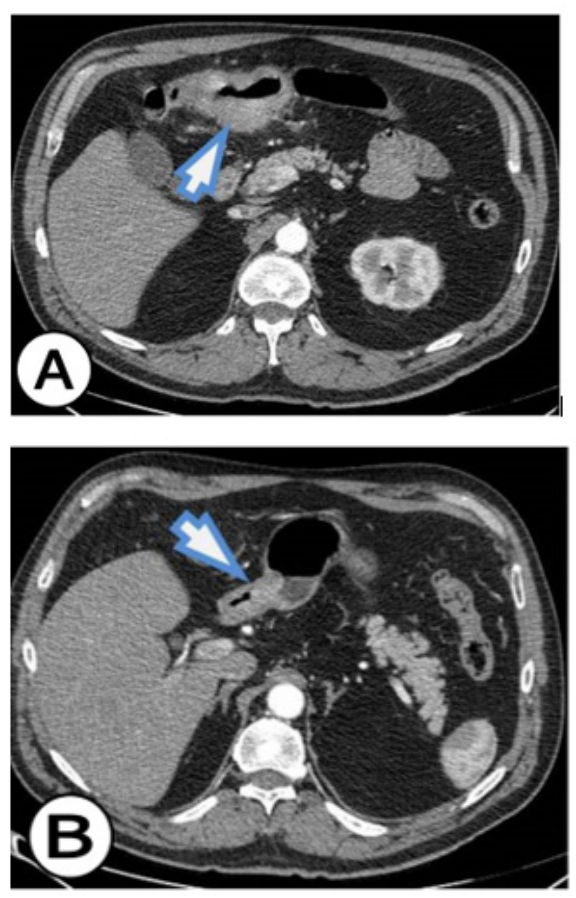

Figure 4: (CT angiography from 22.07.2015). Axial CT images of the abdomen at the level of the transverse colon and the antral part of the stomach. A) Malignant lesion of the transverse colon with narrowing of the lumen is seen (arrow). B) A malignant lesion of the gastric antrum is seen (arrow). 
In the presented case, an extremely fast progression of the malignant process is noticed, which is only 7 months leads to the creation of a block tumor between the antrum of the stomach and the transverse colon. After a CT scan of the abdomen, our initial diagnosis was GIST. But after the analysis of CT angiography made 7 months ago, we think that it is the primary cancer of the colon with a metastatic deposit in the stomach. However, the possibility remains that these are synchronous adenocarcinomas of the colon and adenocarcinoma of the stomach, which is extremely rare. The final resolution of the dilemma is made possible by the pathohistological analysis of the removed abdominal TU mass and immunohistochemically examinations performed with CK20 and CK7. Immunohistochemically studies have shown that CK20 is positive for the tumor population, CK7 is negative for the tumor population corresponding to primary colon cancer, and TU in the stomach is a metastatic deposit. If CK20 and CK7 are positive for the tumor population or CK20 is negative for the tumor population and CK7 is positive for the tumor population, then it may be primary gastric cancer (Figure 4).

In the available literature, we found a case of adenocarcinoma of the colon that metastasized to the stomach. Paulo Moacir de Oliveira Campoli, et al. [6] in their paper cite a case of adenocarcinoma of the colon that metastasized to the stomach. The presence of gastric metastases is a rare condition, and major studies published in the literature are based on endoscopic findings, autopsies, and surgical specimens, or a combination of these three methods. The most commonly described primary cancers that metastasize to the stomach are lung cancer, breast cancer, malignant melanoma, and esophageal cancer [6-8]. Metastasis of malignant processes is very rare in the digestive tract, however, research and autopsy findings indicate a much higher incidence. After all, gastric metastases have been described for certain tumors, such as melanoma, breast cancer, and lung cancer.

Overall, more than $96 \%$ of cases of signet ring cell carcinoma occur in the stomach, with the remaining cases occurring in the colon, rectum, gallbladder, pancreas, bladder, and lungs. The incidence of signet ring cell type cancer in the colon is 0.1 to $2.4 \%$, and clinical features include an advanced stage at diagnosis, large tumor mass, proximal location, young patient, susceptibility to lymph vascular invasion, and peritoneal scattering [9-11]. Synchronous (at the same time) and metachronous (at different time intervals) gastrointestinal (GIT) cancers are extremely rare. In $3-5 \%$ of cases of colorectal cancer, synchronous and metachronous development of two or more primary adenocarcinomas occurs [12]. There are only a few such cases that have been published in the literature [13]. Zubair Ahmad, et al. [14] reported a case of a 41-year-old patient with metachronous carcinoma of the right colon and gastric cancer, stating that they had not encountered such a case in the previously published literature. Synchronous or metachronous adenocarcinoma of the colon and adenocarcinoma of the stomach is extremely rare. The patient was diagnosed with both adenocarcinomas of the colon and adenocarcinoma of the stomach. On the immunohistochemically profile gastric adenocarcinoma in this patient was CK7 positive and CK20 negative; while adenocarcinoma of the colon was CK7 negative and CK20 positive. CK7/CK20 expression for gastric cancer and colon cancer is significantly different. About $70 \%$ of cases of gastric cancer are CK7 positive, and only $20 \%$ are CK20 positive. On the other hand, $95 \%$ of colorectal cancer cases are CK7 negative and CK20 positive. Negative CK7, and positive CK20 profile, in a large percentage, are in favor of colon cancer, while CK7 positive and CK20 negative profile is in favor of metastasis $[15,16]$. In the literature, we find several papers that describe several metachronous and synchronous cancers of the gastrointestinal tract. Iioka, et al. [2] report a triple case of metachronous carcinoma of the sigmoid colon, stomach, and esophagus. Oncel, et al. [8] report a case of a patient with metachronous carcinoma of the stomach, colon, and thyroid. Although patients with multiple cancers are uncommon, they are of particular importance because of the possibility of developing a second or third carcinoma in patients who have already been treated for primary cancer. Mukai, et al. [17] describe a case of a patient who develops triple metachronous cancer of the esophagus, colon, and kidney several years after treatment. Tamura, et al. [13] show a case of triple synchronous cancer of the stomach, colon, and gallbladder in a 70-year-old man. Pricop, et al. [5] report a case of metachronous primary cancer of the colon and stomach in a 69-year-old woman. They also stressed the importance of the possibility of developing metachronous cancer in patients treated for a primary malignant tumor. Another case of synchronous carcinoma of the transverse colon and early gastric cancer has been reported by Nakata, et al. [18]. Parag Brahmbhatta, et al. [19] report a case of recurrent colon adenocarcinoma as duodenal metastasis in a 54-year-old woman who underwent surgery for caecum adenocarcinoma two years ago.

\section{Acknowledgement}

None.

\section{Conflict of Interest}

No conflict of interest.

\section{References}

1. (2007) World Cancer Research Fund and American Institute for Cancer Research Food, Nutrition, Physical Activity, and the Prevention of Cancer: A Global Perspective.Washington, DC: American Institute for Cancer Research.

2. Iioka Y, Tsuchida A, Okubo K, Ogiso M, Ichimiya H, et al. (2000) Metachronous triple cancers of the sigmoid colon, stomach, and esophagus: report of a case. Surg Today 30: 368-371.

3. Green LK (1990) Hematogenous metastases to the stomach. A reviewof 67 cases. Cancer 65: 1596-1600. 
4. Feczko PJ, Collins DD, Mezwa DG (1993) Metastatic disease involving the gastrointestinal tract. Radiol Clin North Am 31: 1359-1373.

5. Pricop C, Lefter LP, Scripcariu V, Danciu M, Buleu D, et al. (2005) Metachronous primary cancers of the colon and stomach. Rev Med Chir Soc Med Nat Iasi 109: 817-821.

6. Paulo Moacir de Oliveira Campoli, Flávio Hayato Ejima, Daniela Medeiros Milhomem Cardoso, Osterno Queiroz da Silva, Jales Benevides Santana Filho, et al. (2006) Metastatic cancer to the stomach. Gastric Cancer 9: 19-25.

7. Lee HC, Yang MT, Lin KY, Tu HY, Zhang TA, et al. (2004) Metastases from gastric carcinoma to colon in the form of multiple flat elevated lesions: a case report. Kaohsiung J Med Sci 20: 552-557.

8. Oncel M, Kurt N, Altuntas YE, Ozturk S, Ozdemir N, et al. (2003) A patient with metachronous gastric, colonic, and thyroid cancers: a case report Int Surg 88: 1-5.

9. Sim HL, Tan KY, Poon PL, Cheng A (2008) Primary rectal signet ring cell carcinoma with peritoneal dissemination and gastric secondaries. World J Gastroenterol 14: 2118-2120.

10. Tung SY, Wu CS, Chen PC (1996) Primary signet ring cell carcinoma of colorectum: an age- and sex-matched controlled study. Am J Gastroenterol 91: 2195-2199.

11. Jinn-Shiun Chen, Pao-Shiu Hsieh, Jy-Ming Chiang, Chien-Yuh Yeh, WenSy Tsai, et al (2010) Clinical outcome of signet ring cell carcinoma and mucinous adenocarcinoma of the colon. Chang Gung Med J 33: 51-57.

12. Paulo Moacir de Oliveira Campoli, Flávio Hayato Ejima, Daniela Medeiros Milhomem Cardoso, Osterno Queiroz da Silva, Jales Benevides Santana Filho, et al. (2006) Metastatic cancer to the stomach Gastric Cancer 9: 19-25.
13. Tamura M, Shinagawa M, Funaki Y (2003) Synchronous triple early cancers occurring in the stomach, colon and gallbladder. Asian J Surg 26: 46-49.

14. Zubair Ahmad, Ayesha Memon, Khurram Minhas (2008) Metachronous Primary Cancers of Colon and Stomach. J Coll Physicians Surg Pak 18(2): 118-120.

15. Lagendijk JH, Mullink H, Van Diest PJ, Meijer GA, Meijer CJ (1998) Tracing the origin of adenocarcinomas with unknown primary using immunohistochemistry: differential diagnosis between colonic and ovarian carcinomas as primary sites. Hum Pathol 29: 491-497.

16. Park SY, Kim HS, Hong EK, Kim WH (2002) Expression of cytokeratins 7 and 20 in primary carcinomas of the stomach and colorectum and their value in the differential diagnosis of metastatic carcinomas to the ovary. Hum Pathol 33: 1078-1085.

17. Mukai M, Makuuchi H, Mukohyama S, Oida Y, Himeno S, et al. (2001) Quintuple carcinomas with metachronous triple cancer of the esophagus, kidney and colonic conduit following synchronous double cancer of the stomach and duodenum. Oncol Rep 8: 111-114.

18. Nakata Y, Kimura K, Tomioka N, Kawasaki S, Takagaki Y (1999) Successful simultaneous operation of concomitant early gastric cancer, transverse colon cancer, and a common iliac artery aneurysm. Surg Today 29: 782784.

19. Parag Brahmbhatt, Jason Ross, Atif Saleem, Jason McKinney, Pranav Patel, et al. (2013) Recurrent Adenocarcinoma of Colon Presenting as Duodenale Metastasis With Partial Gastric Outlet Obstruction: A Case Report With Review of Literature. World J Oncol 4(2): 102-106. 